\title{
Ultrasound-Guided Laser-Induced Thermal Therapy for Small Palpable Invasive Breast Carcinomas: A Feasibility Study
}

\author{
S. van Esser, $\mathrm{MD}^{1}$, G. Stapper, $\mathrm{MD}^{2}$, P. J. van Diest, $\mathrm{PhD}^{3}$, M. A. A. J. van den Bosch, $\mathrm{PhD}^{2}$, J. H. G. M. Klaessens, \\ $\mathrm{MSc}^{4}$, W. P. Th. M. Mali, $\mathrm{PhD}^{2}$, I. H. M. Borel Rinkes, $\mathrm{PhD}^{1}$, and R. van Hillegersberg, $\mathrm{PhD}^{1}$ \\ ${ }^{1}$ Department of Surgical Oncology, University Medical Center Utrecht, Utrecht, The Netherlands; ${ }^{2}$ Department of \\ Radiology, Radiotherapy and Nuclear Medicine, University Medical Centre Utrecht, Utrecht, The Netherlands; \\ ${ }^{3}$ Department of Pathology, University Medical Centre Utrecht, Utrecht, The Netherlands; ${ }^{4}$ Department of Clinical Physics, \\ University Medical Centre Utrecht, Utrecht, The Netherlands
}

\begin{abstract}
Background. The next step in breast-conserving surgery for small breast carcinomas could be local ablation. In this study, the feasibility of ultrasound-guided laser-induced thermal therapy (LITT) is evaluated.

Methods. Patients with large-core needle biopsy-proven invasive, palpable breast carcinoma (clinically $\leq 2 \mathrm{~cm}$ ) underwent ultrasound-guided LITT, followed by surgical excision. Completeness of ablation was determined by both hematoxylin and eosin staining and nicotinamide adenosine diaphorase staining.

Results. Fourteen patients completed the treatment. The mean histological tumor size was $17 \mathrm{~mm}$ (range, 8$37 \mathrm{~mm}$ ); 6 of 14 tumors were histologically larger than the clinical entry threshold of $2 \mathrm{~cm}$. The power applied in all patients was $7 \mathrm{~W}$, and the mean treatment time was 21.4 min (range, 15-30 min). In one patient, a skin burn occurred, and one patient had a localized pneumothorax that could be treated conservatively. In 7 (50\%) of 14 patients, the tumor was completely ablated, as confirmed by nicotinamide adenosine diaphorase staining. In 11 cases, extensive in-situ carcinoma was present. In one case, the in-situ carcinoma was also completely ablated. A total of seven (88\%) of eight tumors $<2 \mathrm{~cm}$ in size were completely ablated versus one $(17 \%)$ of six tumors that were $\geq 2 \mathrm{~cm}$ in size $(P=.026)$.
\end{abstract}

Conclusions. Successful LITT of invasive breast cancer seems to be feasible when confined to small $(<2 \mathrm{~cm})$

(C) The Author(s) 2009. This article is published with open access at Springerlink.com

First Received: 7 April 2009;

Published Online: 9 June 2009

S. van Esser, MD

e-mail: s.vanesser@umcutrecht.nl nonlobular carcinomas without surrounding extensive insitu component and angioinvasion. However, to implement LITT in a curative setting, improvements in imaging to more reliably preoperatively assess tumor size and monitoring of fiber tip placement and treatment affect are essential.

Over the past decades, there has been a transition from extensive oncological surgery to minimally invasive oncological surgery. In the treatment of breast carcinomas, clearly the transition from the radical Halsted operation to breastconserving surgery (BCS) and adjuvant radiotherapy for the small carcinomas was a great improvement. ${ }^{1-3}$ The introduction of the sentinel node procedure is in line with the aim at minimally invasive surgery. ${ }^{4}$ As a result of the extensive screening programs for breast cancer, the proportion of small breast carcinomas has increased. ${ }^{5}$ Especially for this group of patients with early breast cancer, there is still room for improvement of the treatment. The cosmetic outcome of BCS is often disappointing, and BCS carries a relatively high morbidity rate mainly due to postoperative bleeding (up to $11 \%$ ) and infections (3\% to $4 \%){ }^{6-8}$ As a result of surgical complications, adjuvant treatment may be delayed, affecting the outcome unfavorably. ${ }^{9}$

The next step in BCS could be local ablation of small breast carcinomas, provided it is equally effective but with fewer complications and a better cosmetic outcome than a lumpectomy. Local ablation should efficiently and completely destroy the invasive breast carcinoma and surrounding in-situ carcinoma. Currently, cryosurgery, radiofrequency ablation, laser-induced thermal therapy (LITT), microwave ablation, and high-intensity focused ultrasound ablation are clinically available local ablation 
modalities. ${ }^{10-15}$ In all of these modalities, a change in temperature is used to lethally damage the intracellular DNA binding structures, thereby causing cell death. ${ }^{16,17}$ LITT requires a laser fiber to guide the light energy directly into the tissue to be treated. Upon absorption in the tissue, heat is produced, inducing lethal thermal injury. At present, LITT and radiofrequency ablation are mostly used to treat unresectable colorectal liver metastases as an alternative to surgery. ${ }^{18,19}$ The results are promising, and possibly in the future, these modalities could also be used for the local ablation of breast carcinoma in a curative setting.

To implement this minimally invasive approach, several steps need to be taken. First, the exact tumor size should be reliably assessed. Second, the treatment should be safe and able to completely destroy all tumor tissue (including insitu cancer) locally. Finally, a reliable real-time way to monitor the treatment results should be available.

In this feasibility study, we evaluated the second prerequisite by ultrasound-guided LITT for local treatment of patients with clinically small palpable breast carcinomas.

\section{MATERIALS AND METHODS}

The study was approved by the medical ethical committee of our hospital. All patients had a palpable invasive breast carcinoma diagnosed by an ultrasound-guided largecore needle biopsy (LCNB). The tumor characteristics (i.e., grade, estrogen receptor status, progesterone receptor status, HER-2/neu status, and mitotic activity index) were preoperatively determined on the LCNB.

Inclusion criteria were age $>18$ years, palpable cT1 (as measured by preoperative ultrasound and mammography), unifocal invasive breast carcinoma, tumor visible on ultrasound, preoperative tumor characteristics available, and distance of $\geq 1 \mathrm{~cm}$ from the thoracic wall and the overlying skin.

A sentinel lymph node biopsy (SLNB) was performed before the LITT procedure. For the SLNB, four depots of a total of $120 \mathrm{MBq}{ }^{99 \mathrm{~m}} \mathrm{Tc}$ nanocolloid were injected peritumorally, and static images were acquired up to $4 \mathrm{~h}$ after injection. Peroperatively, $1 \mathrm{~mL}$ of patent blue (Bleu patenté V "Guerbet") was injected peritumorally. The exact tumor size was measured in two dimensions after the SLNB by ultrasound (Philips, iU22 scanner, equipped with an $8 \mathrm{MHz}$ linear array transducer).

Next, a 17-gauge guidance needle (Bard Truguide, Covington, United Kingdom) was placed into the center of the tumor. The position of the needle was checked ultrasonographically in three dimensions. Both the tumor size assessment and the placement of the guidance needle were performed by a dedicated breast radiologist.
Next, an uncooled Microdom LITT laser fiber with an active length of $2.5 \mathrm{~cm}$ was inserted into the tumor and attached to an Nd:YAG continuous laser of $1045 \mathrm{~nm}$ (KLS Martin, Umkirch, Germany). After confirming the exact position, the guidance needle was retracted to avoid heath conduction to the skin and the procedure commenced. The amount of energy needed to fully ablate the tumor was determined in an experimental ex vivo setting in bovine udder tissue. The maximum tumor size determined the amount of energy that was used.

After the ablation, a wide local excision or mastectomy was performed according to local practice.

At pathology, the margins of the specimen were inked and the specimen was sliced in 5-mm slices. The macroscopic lesion size was recorded, and samples were taken from the center of the coagulated area and the transition zone between tumor and tumor-free margin (Fig. 1). The samples were snap frozen and embedded in paraffin. Both hematoxylin and eosin staining of paraffin-embedded sections and nicotinamide dinucleotide adenosine diaphorase (NADH) staining of the frozen sections were used to determine tumor vitality after ablation. Participating patients underwent routine outpatient follow-up and standard breast radiotherapy as a part of BCS. On the basis of the status of the sentinel node and primary tumor characteristics, patients underwent an additional axillary dissection and/or adjuvant chemotherapy.

\section{RESULTS}

Fourteen patients completed the LITT procedure. Baseline characteristics are summarized in Table 1.

Most of the tumors (50\%) were located in the upper lateral quadrant of the breast, and 12-14 tumors were invasive ductal carcinomas. All patients underwent a SLNB before ablation; in 13 (93\%) of 14, the sentinel node was found. Six patients had axillary metastases, with isolated tumor cells in

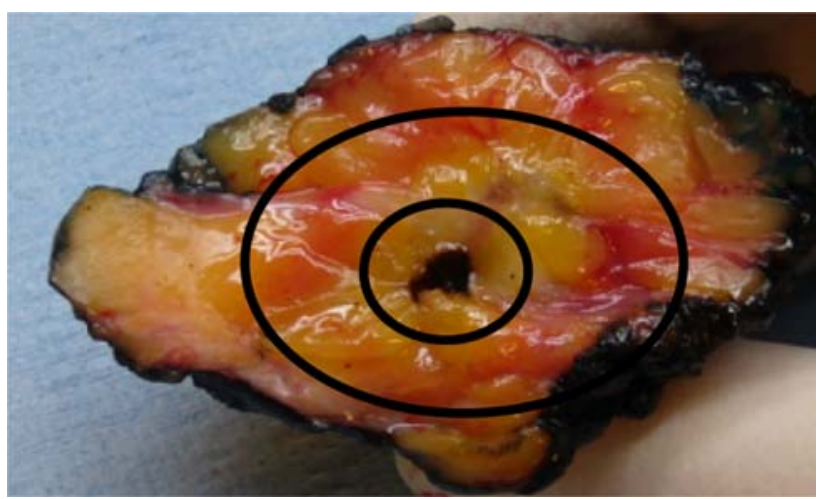

FIG. 1 Macroscopic view of ablated tumor tissue. Outer circle indicates the ablation zone; inner circle shows fiber track 
TABLE 1 Baseline patient characteristics

\begin{tabular}{lc}
\hline Characteristic & Value \\
\hline Median age (y) (range) & $54.5(35-85)$ \\
Median ultrasound tumor size (mm) (range) & $14.5(9-20)$ \\
Median histological tumor size (mm) (range) & $17(8-37)$ \\
Tumor location, $n(\%)$ & \\
$\quad$ Upper lateral & $7(50)$ \\
$\quad$ Lower lateral & $3(22)$ \\
$\quad$ Lower medial & $2(14)$ \\
$\quad$ Retroareolar & $2(14)$ \\
Tumor histology, $n(\%)$ & \\
$\quad$ Ductal carcinoma & $12(86)$ \\
$\quad$ Lobular carcinoma & $1(7)$ \\
$\quad$ Ductolobular carcinoma & $1(7)$ \\
SLNB found tumor, $n(\%)$ & $13(93)$ \\
SLNB free of tumor, $n(\%)$ & $7(50)$ \\
Breast-conserving surgery, $n(\%)$ & $13(93)$ \\
Mastectomy, $n(\%)$ & $1(7)$ \\
\hline
\end{tabular}

SLNB sentinel lymph node biopsy

three and macrometastases in the other three. Two of the patients with axillary isolated tumor cells chose not to undergo an additional axillary dissection. The three patients with macrometastases all underwent additional axillary dissection.

LITT time ranged from 15 to $30 \mathrm{~min}$ (mean, $21.4 \mathrm{~min}$ ). The power applied was $7 \mathrm{~W}(2.8 \mathrm{~W} / \mathrm{cm}$ diffuser length $)$ in all cases. The mean ultrasound-measured tumor size was $15.3 \mathrm{~mm}$, and the median ultrasound-measured lesion size (Figs. 1 and 2) after ablation was $18 \mathrm{~mm}$ (range, 9-22 $\mathrm{mm}$ ). In all patients, extensive air bubble formation around the fiber tip was observed on ultrasound. The maximum diameter of the largest histological lesion size was $30 \mathrm{~mm}$. The

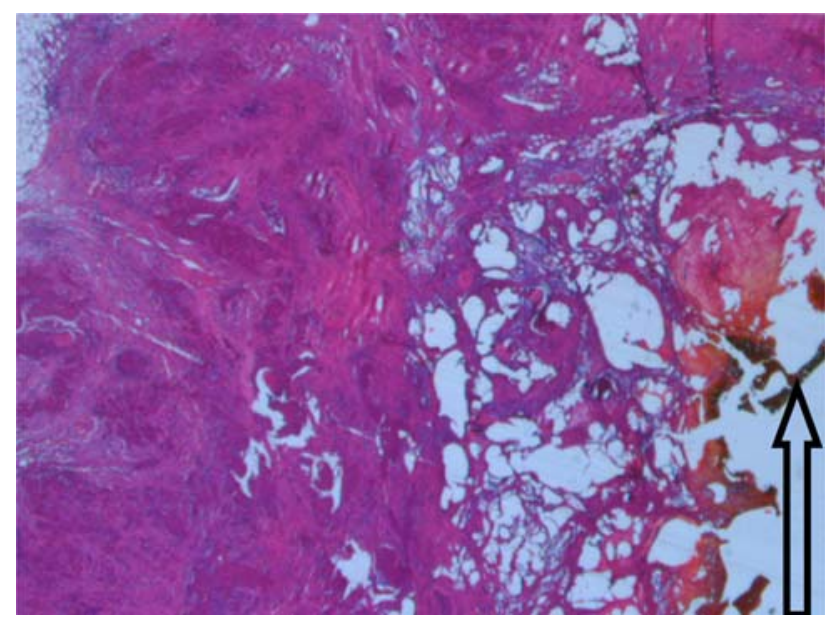

FIG. 2 Microscopic view of ablation zone (hematoxylin and eosin; original magnification, $\times 25)$. Arrow indicates position of laserinduced thermal therapy probe median histological tumor diameter was $17 \mathrm{~mm}$ (range, 8-37 mm), and the median histological ablated tumor diameter was $23 \mathrm{~mm}$ (range, 2-35 mm). In total, 7 (50\%) of 14 of the invasive tumors were completely ablated, as confirmed by NADH staining. In 11 cases, an extensive in-situ component surrounded the tumor, and in 1 case, this in-situ component was also completely ablated. In 2 (14\%) of 14 cases, only a very small component of the tumor seemed to be ablated as a result of misplacement of the guidance needle. Two tumors had an extensive angioinvasive growth pattern, resulting in preoperative underestimation of tumor size.

The invasive carcinomas that were histologically $<2 \mathrm{~cm}$ in size were significantly more frequent completely ablated ( 7 of $8,88 \%$ ) than the invasive carcinomas that were $>2 \mathrm{~cm}$ in size ( 1 of $6,17 \%)(P=.026)$.

In one patient, a superficial skin burn occurred during the procedure. This was treated with a partial skin resection as a part of the lumpectomy. In another patient, the guidance needle was placed close to the pectoral muscle, resulting in a localized pneumothorax that could be treated conservatively.

All but one patient had subsequent BCS. One patient preferred breast amputation to avoid radiotherapy; this patient experienced postoperative bleeding that could be treated conservatively. A postoperative wound infection occurred in two patients.

\section{DISCUSSION}

The aim of this study was to assess the feasibility of ultrasound-guided LITT in invasive breast cancers clinically up to $2 \mathrm{~cm}$ in size. Overall, the invasive breast cancer was completely ablated in $7(50 \%)$ of 14 patients. There was a clear association between the success rate of LITT and tumor size: cancers were completely ablated in seven (88\%) of eight and one $(17 \%)$ of six of patients with a carcinoma $<2 \mathrm{~cm}$ and $\geq 2 \mathrm{~cm}$, respectively. Our results thereby show that LITT can completely ablate small invasive breast carcinomas.

Previous studies have confirmed the importance of tumor size in completely ablating breast carcinomas. ${ }^{15,20}$ There were three reasons for the discrepancies between the clinically/radiologically estimated tumor size and the histological tumor size. First, two tumors showed a lobular growth pattern. It is well known that tumor size for this type of tumor is more difficult to assess, and often this type of tumor is histologically more extensive than estimated as a result of their diffuse growth pattern. ${ }^{21}$ In our opinion, tumors showing lobular differentiation should therefore be excluded from local ablation treatment in a curative setting. Second, in 10 of 14 cases, extensive ductal carcinoma-insitu (DCIS) was present outside the ablation zone. 
Incompletely ablated DCIS may later well give rise to an invasive recurrence, although radiotherapy helps to control this. Invasive carcinomas showing extensive DCIS on LCNB should be excluded from local ablation in a curative setting as well. Third, in two patients, extensive angioinvasive growth was present, which was not seen on the LCNB in one patient. These growth patterns cannot be detected by mammography and/or ultrasound. ${ }^{22-25}$ Therefore, to arrive at successful LITT, preoperative tumor size assessment needs to be improved. To this end, magnetic resonance imaging (MRI) is likely to be the first option, although optical imaging may prove to be important in the future. $^{26,27}$

To implement LITT as the breast-conserving treatment for small carcinomas of the breast, reliable intraoperative imaging is also needed during the LITT procedure to monitor placement of the laser fiber and the effect of treatment. The importance of monitoring the placement fiber is emphasized by an example: in one patient, a localized pneumothorax occurred as a result of misplacement of the guidance needle. Pneumothorax is a known complication after LCNB, but so far, it is has not been described as a complication of minimally invasive treatment of breast carcinoma. ${ }^{28}$ Finally, adequate imaging is vital to the treatment monitoring. In most of the studies performed on LITT of breast carcinomas, the treatment parameters were predetermined. ${ }^{11,29}$ When treatment parameters are predetermined, possible cooling effects of blood flow and differences in density of breast and tumor tissue cannot be taken into account, possibly leading to undertreatment of the tumor tissue. Real-time monitoring and adjusting of the treatment parameters would be ideal. In this study, the progress of the ablation was monitored by ultrasound. Temperatures close to the fiber tip reach $100^{\circ} \mathrm{C}$, causing evaporation of the tumor tissue (Fig. 2). ${ }^{29,30}$ This evaporation causes hyperechoic air bubbles, making ultrasound monitoring difficult. ${ }^{31}$ Nevertheless, the temperature in the fatty tissue surrounding the tumor increases slowly and is lower, possibly resulting in less fatty necrosis and therefore a better cosmetic outcome and less reinterventions. ${ }^{32}$ Although not clinically available yet, real-time magnetic resonance-guided thermal mapping is a promising new technique that could become a good alternative to ultrasound. $^{33}$ Because LITT causes ablation through the absorption of light energy, it can be monitored by MRI, whereas radiofrequency ablation is based on radiofrequent waves, causing artifacts in MRI that make MRI monitoring a challenge. $^{34}$

Determination of the sentinel node via SLNB is important for adequate staging and further treatment. Possibly local ablation of the tumor tissue disturbs the lymph drainage pattern. Therefore, in this study, the SLNB was performed directly before the LITT procedure. In 13 $(93 \%)$ of 14 patients, the sentinel node was detected and collected - a rate comparable to that described in the current literature. ${ }^{35}$ In available studies on LITT to date, no clear data have been provided on the timing of the SLNB.

Besides the pneumothorax in one patient, another patient had a skin burn that could easily be treated. Other complications described in literature, such as gaseous rupture of tumor tissue and hyperemia, were not observed in this feasibility study. ${ }^{11,15,30}$

The NADH staining confirmed the hematoxylin and eosin staining results in all cases. As has been shown in other studies, NADH is a reliable stain to confirm tissue vitality. NADH staining requires tissue to be snap frozen, which can be impractical in a routine clinical setting. Cytokeratin $8 / 18$ could be a good and practical alternative because it does not requires tissue to be snap frozen. ${ }^{10}$

In conclusion, successful LITT of invasive breast cancer seems to be feasible when confined to small $(<2 \mathrm{~cm})$ nonlobular carcinomas without a surrounding extensive insitu component and angioinvasion. However, to implement LITT in a curative setting, it will be essential to improve imaging to more reliably preoperatively assess tumor size and to monitor fiber tip placement and treatment effect.

OPEN ACCESS This article is distributed under the terms of the Creative Commons Attribution Noncommercial License which permits any noncommercial use, distribution, and reproduction in any medium, provided the original author(s) and source are credited.

\section{REFERENCES}

1. Fisher B, Redmond C, Poisson R, et al. Eight-year results of a randomized clinical trial comparing total mastectomy and lumpectomy with or without irradiation in the treatment of breast cancer. N Engl J Med. 1989;320:822-8.

2. Mann BA, Samet JM, Hunt WC, et al. Changing treatment of breast cancer in New Mexico from 1969 through 1985. JAMA. 1988;259:3413-7.

3. Veronesi U, Cascinelli N, Mariani L, et al. Twenty-year followup of a randomized study comparing breast-conserving surgery with radical mastectomy for early breast cancer. $N$ Engl $\mathrm{J} \mathrm{Med}$. 2002;347:1227-32.

4. Borgstein PJ, Pijpers R, Comans EF, et al. Sentinel lymph node biopsy in breast cancer: guidelines and pitfalls of lymphoscintigraphy and gamma probe detection. J Am Coll Surg. 1998;186: 275-83.

5. Anttinen J, Kautiainen H, Kuopio T. Role of mammography screening as a predictor of survival in postmenopausal breast cancer patients. Br J Cancer. 2006;94:147-51.

6. Bakker XR, Roumen RM. Bleeding after excision of breast lumps. Eur J Surg. 2002;168:401-3.

7. El-Tamer MB, Ward BM, Schifftner T, et al. Morbidity and mortality following breast cancer surgery in women: national benchmarks for standards of care. Ann Surg. 2007;245:665-71.

8. Vilar-Compte D, Jacquemin B, Robles-Vidal C, Volkow P. Surgical site infections in breast surgery: case-control study. World $J$ Surg. 2004;28:242-6.

9. Furey PC, Macgillivray DC, Castiglione CL, Allen L. Wound complications in patients receiving adjuvant chemotherapy after mastectomy and immediate breast reconstruction for breast cancer. J Surg Oncol. 1994;55:194-7. 
10. Burak WE Jr, Agnese DM, Povoski SP, et al. Radiofrequency ablation of invasive breast carcinoma followed by delayed surgical excision. Cancer. 2003;98:1369-76.

11. Dowlatshahi K, Francescatti DS, Bloom KJ. Laser therapy for small breast cancers. Am J Surg. 2002;184:359-63.

12. Furusawa H, Namba K, Thomsen $S$, et al. Magnetic resonanceguided focused ultrasound surgery of breast cancer: reliability and effectiveness. J Am Coll Surg. 2006;203:54-63.

13. Gardner RA, Vargas HI, Block JB, et al. Focused microwave phased array thermotherapy for primary breast cancer. Ann Surg Oncol. 2002;9:326-32.

14. Sabel MS, Kaufman CS, Whitworth P, et al. Cryoablation of early-stage breast cancer: work-in-progress report of a multiinstitutional trial. Ann Surg Oncol. 2004;11:542-9.

15. van Esser S, van den Bosch MA, van Diest PJ, et al. Minimally invasive ablative therapies for invasive breast carcinomas: an overview of current literature. World J Surg. 2007;31:2284-92.

16. Heisterkamp J, van Hillegersberg R, Ijzermans JN. Critical temperature and heating time for coagulation damage: implications for interstitial laser coagulation (ILC) of tumors. Lasers Surg Med. 1999;25:257-62.

17. Heisterkamp J, van Hillegersberg R, Zondervan PE, Ijzermans JN. Metabolic activity and DNA integrity in human hepatic metastases after interstitial laser coagulation (ILC). Lasers Surg Med. 2001;28:80-6.

18. Veenendaal LM, Borel Rinkes IH, van Hillegersberg R. Multipolar radiofrequency ablation of large hepatic metastases of endocrine tumours. Eur J Gastroenterol Hepatol. 2006;18:89-92.

19. Vogl TJ, Naguib NN, Eichler K, et al. Volumetric evaluation of liver metastases after thermal ablation: long-term results following MR-guided laser-induced thermotherapy. Radiology. 2008;249: 865-71.

20. Medina-Franco H, Soto-Germes S, Ulloa-Gómez JL, et al. Radiofrequency ablation of invasive breast carcinomas: a phase II trial. Ann Surg Oncol. 2008;15:1689-95.

21. Lopez JK, Bassett LW. Invasive lobular carcinoma of the breast: spectrum of mammographic, US, and MR imaging findings. Radiographics. 2009;29:165-76.

22. Dummin LJ, Cox M, Plant L. Prediction of breast tumor size by mammography and sonography - a breast screen experience. Breast. 2007;16:38-46.

23. Berg WA, Gutierrez L, NessAiver MS, et al. Diagnostic accuracy of mammography, clinical examination, US, and MR imaging in preoperative assessment of breast cancer. Radiology. 2004;233: 830-49.

24. Golshan M, Fung BB, Wiley E, et al. Prediction of breast cancer size by ultrasound, mammography and core biopsy. Breast. 2004; 13:265-71.

25. Shoma A, Moutamed A, Ameen M, Abdelwahab A. Ultrasound for accurate measurement of invasive breast cancer tumor size. Breast J. 2006;12:252-6.

26. Schouten Velden AP, Boetes C, Bult P, Wobbes T. The value of magnetic resonance imaging in diagnosis and size assessment of in situ and small invasive breast carcinoma. Am J Surg. 2006; 192:172-8.

27. van der Ven S, Elias SG, Wiethoff AJ, et al. Diffuse optical tomography of the breast: preliminary findings of a new prototype and comparison with magnetic resonance imaging. Eur Radiol. 2009;19(5):1108-13.

28. Meyer JE, Smith DN, Lester SC, et al. Large-core needle biopsy of nonpalpable breast lesions. JAMA. 1999;281:1638-41.

29. Bloom KJ, Dowlat K, Assad L. Pathologic changes after interstitial laser therapy of infiltrating breast carcinoma. Am J Surg. 2001;182:384-8.

30. Akimov AB, Seregin VE, Rusanov KV, et al. Nd:YAG interstitial laser thermotherapy in the treatment of breast cancer. Lasers Surg Med. 1998;22:257-67.

31. Varghese T, Techavipoo U, Zagzebski JA, Lee FT Jr. Impact of gas bubbles generated during interstitial ablation on elastographic depiction of in vitro thermal lesions. J Ultrasound Med. 2004;23: 535-44.

32. Lövey K, Fodor J, Major T, et al. Fat necrosis after partial-breast irradiation with brachytherapy or electron irradiation versus standard whole-breast radiotherapy-4-year results of a randomized trial. Int J Radiat Oncol Biol Phys. 2007;69:724-31.

33. Rieke V, Butts Pauly K. MR thermometry. J Magn Reson Imaging. 2008;27:376-90.

34. van den Bosch M, Daniel B, Rieke V, et al. MRI-guided radiofrequency ablation of breast cancer: preliminary clinical experience. $J$ Magn Reson Imaging. 2008;27:204-8.

35. Bergkvist L, Frisell J, Swedish Breast Cancer Group, Swedish Society of Breast Surgeons. Multicentre validation study of sentinel node biopsy for staging in breast cancer. Br J Surg. 2005; 92:1221-4. 